\title{
Identification of Very Low Frequency (VLF) Whistlers transients using Feed Forward Neural Network (FFNN)
}

\author{
D.K. Sondhiya ${ }^{1}$, S.K. Kasde ${ }^{2}$, Dishansh Raj Upwar ${ }^{3}$, A.K. Gwal ${ }^{4}$ \\ ${ }^{I}$ Department of Physics, Lakshmi Narain College of Technology\&Science, Bhopal, India \\ ${ }^{2}$ Department of Physics, Barkatullah University, Bhopal, India \\ ${ }^{3}$ Department of Physics, Oriental Institute of Science and Technology, Bhopal, India \\ ${ }^{4}$ AISECT University, Bhopal India
}

\begin{abstract}
The automatic identification of VLF whistler transients is an important practical goal for ionospheric and magnetospheric science because they give useful information regarding propagating medium particularly of the plasmasphere. We have developed a neural network based system to identify four types of whistlers (i.e diffuse, dispersive, multipath and spicky) recorded by DEMETER (Detection of electromagnetic emission form earthquake region) satellite. Wavelet transform is applied to extract the characteristics features of whistlers which are used to train the Feed Forward Neural Network (FFNN). The data required to train the network were collected from two year (2008-2010) observations of DEMETER satellite. The results show that the proposed FFNN can accurately identify the whistler transients.
\end{abstract}

Keywords:Ionosphere, Wavelet transform, Feature extraction, Neural Network, VLF Whistlers

\section{Introduction}

Whistlers are fast and short duration burst of Very Low Frequency (VLF, 3-30 kHz) electromagnetic waves generated by lightning return strokes which can be recorded by ground receivers and by satellites [1]. They have been utilized as diagnostic tool for the magnetosphere $[2,3,4]$. Whistlers and other VLF transients such as hiss and chorus propagating in magnetosphere are useful tools to examine the ionosphere and magnetosphere [5,6]. The accurate spectral analysis of whistlers enables us to study the behavior of electromagnetic emissions and their propagation in ionosphere and magnetosphere. From the last few decades whistlers transient have also been used as earthquake precursor [7], therefore, proper accurate identification of whistlers is very important. Whistlers are difficult to detect because of their short duration. The main distinguishing feature of whistlers is their frequency- time structure. The instrument for measuring the whistlers should have high sampling rate to provide greater accuracy in terms of amplitude and frequency content. Blecki et al. [8] characterized the disturbances in Extremely Low Frequency (up to $1250 \mathrm{~Hz}$ ) signals observed by DEMETER satellite through Wavelet and Bispectral analyses. Numerous other techniques of signal analysis have been used to obtain various features of signals. In the geophysical domain, it has been used for waveform recognition and first-break [9], for electromagnetic [10] and seismic inversion purposes [11, 12] seismic deconvolution[13] and event classification [14].

Romeo et al. [15] proposed a Neural Network (NN) based system for recognizing different types of earthquakes or in detecting the presence of a seismic event. Schmitter et al. [16] suggested that features based on Wavelet and Statistical methods are efficient for analysis and characterization of transients using NN based system. Waibel et al. [17] adopted a Time Delay Neural Network (TDNN) for the whistler phenomena recognition. Buzzi et al. [18] presented an architecture considering several NN, each of which had only two classes to identity. This neural network is very feasible because it allows large variation in each class, explored several time resolution for the detection of one event and estimates the dispersion and amplitude parameter outside the neural network. Popova et al. [19] used neural network for the analysis of VLF/LF signals.

Main aim of this paper is to propose a novel identification approach based on Feed Forward Neural Network (FFNN)to identify four different types of whistlers (i.e., spiky, dispersive, multipath and diffuse). The detection was simplified by extracting various characteristics of whistlers using Statistical and Wavelet based techniques. The main advantages for using FFNN based system for whistler identification are:

- Once trained on the ground the neural network requires minimal and constant computing time, which permits real-time classification of Whistlers.

- The same architecture may be used for classification of others VLF signal transients such as Hiss and Chorus. 
- Neural network accept large variation within a class of phenomena, which allows us to cope with many geophysical parameters that may affect a whistler, altitude of the point of observation invariant latitude, local time and geophysical activity.

\section{Whistler feature extraction}

The goal of the proposed FFNN is to design an algorithm which is capable to identify four types of whistlers. Previous studies [20,21] suggested several important simplifying assumptions and discriminating features of particular class of whistlers:

- Whistlers are highly non-stationary in natures, even same kinds of whistlers itself shows slightly different features.

- The proper grouping of whistlers requires an insensitivity to the time alignment of the transient within the selected time window

In order to accommodate and exploit the characteristics features of whistlers an algorithm was designed. In this study, widely used and most successful features based on time and the frequency domain was evaluated form Wavelet transform [22]. The decomposition levels are chosen such that those parts of the signal that correlate well with the frequencies required for the identification of the whistlers are retained in the Wavelet coefficients. In this work Daubechies wavelet filters ( $\mathrm{db} 4)$ with number of decomposition levels 4 have been used. Thus, whistlers' signals were decomposed into four details coefficients (i.e $D_{1}-D_{4}$ ) and one final approximation coefficient $A_{4}$. Each sub-signal contains transient information within different frequency bands. The block diagram for feature extraction algorithm is illustrated in Fig. 1.

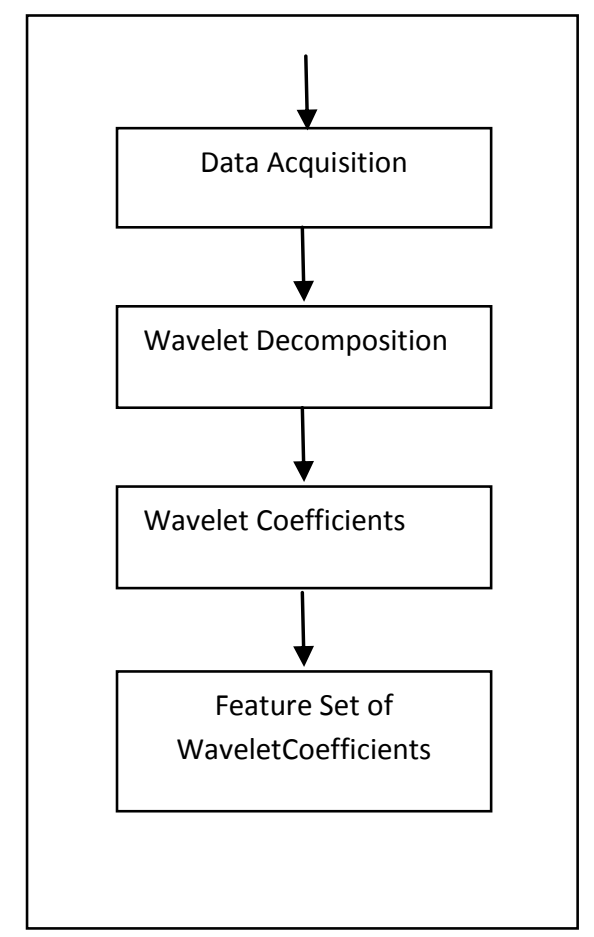

Fig. 1. Whistler feature extraction algorithm.

\section{Wavelet features extraction vector (coefficients extraction)}

The computed details and approximation Wavelet coefficients were used to construct features vector representing the transients. The feature vector for a particular class of whistlers is represented by equation (1) and semantic of common input vector was given in Table 1. It shows the characteristics features of whistler extracted form proposed feature extraction method. 


$$
F_{1}=\left[\begin{array}{c}
P_{1} \\
P_{2} \\
P_{3} \\
P_{4} \\
\vdots \\
P_{31} \\
P_{32}
\end{array}\right]
$$

Table1.Semantic of Common Input Vector

\begin{tabular}{|c|c|}
\hline Vector Elements & Description \\
\hline$P_{1}$ & Mean of Wavelet detail $d_{1}$ coefficient \\
\hline$P_{2}$ & Mean of Wavelet detail $d_{2}$ coefficient \\
\hline$P_{3}$ & Mean of Wavelet detail $d_{3}$ coefficient \\
\hline$P_{4}$ & Mean of Wavelet detail $d_{4}$ coefficient \\
\hline$P_{5}$ & Mean of Wavelet Approximation $A_{4}$ coefficient \\
\hline$P_{6}$ & Standard deviation of Wavelet detail $d_{1}$ coefficient \\
\hline$P_{7}$ & Standard deviation of Wavelet detail $d_{2}$ coefficient \\
\hline$P_{8}$ & Standard deviation of Wavelet detail $d_{3}$ coefficient \\
\hline$P_{9}$ & Standard deviation of Wavelet detail $d_{4}$ coefficient \\
\hline$P_{10}$ & Standard deviation of Wavelet Approximation $A_{4}$ coefficient \\
\hline$P_{11}$ & Maximum of Wavelet detail $d_{1}$ coefficient \\
\hline$P_{12}$ & Maximum of Wavelet detail $d_{2}$ coefficient \\
\hline$P_{13}$ & Maximum of Wavelet detail $d_{3}$ coefficient \\
\hline$P_{14}$ & Maximum of Wavelet detail $d_{4}$ coefficient \\
\hline$P_{15}$ & Maximum of Wavelet Approximation $A_{4}$ coefficient \\
\hline$P_{16}$ & Minimum of Wavelet detail $d_{1}$ coefficient \\
\hline$P_{17}$ & Minimum of Wavelet detail $d_{2}$ coefficient \\
\hline$P_{18}$ & Minimum of Wavelet detail $d_{3}$ coefficient \\
\hline$P_{19}$ & Minimum of Wavelet detail $d_{4}$ coefficient \\
\hline$P_{20}$ & Minimum of Wavelet Approximation $A_{4}$ coefficient \\
\hline$P_{21}$ & Energy of Wavelet detail $d_{1}$ coefficient \\
\hline$P_{22}$ & Energy of Wavelet detail $d_{2}$ coefficient \\
\hline$P_{23}$ & Energy of Wavelet detail $d_{3}$ coefficient \\
\hline$P_{24}$ & Energy of Wavelet detail $d_{4}$ coefficient \\
\hline$P_{25}$ & Entropy of Wavelet detail $d_{1}$ coefficient \\
\hline$P_{26}$ & Entropy of Wavelet detail $d_{2}$ coefficient \\
\hline$P_{27}$ & Entropy of Wavelet detail $d_{3}$ coefficient \\
\hline$P_{28}$ & Entropy of Wavelet detail $d_{4}$ coefficient \\
\hline$P_{29}^{20}$ & Noise of Wavelet detail $d_{1}$ coefficient \\
\hline$P_{30}$ & Noise of Wavelet detail $d_{2}$ coefficient \\
\hline$P_{31}$ & Noise of Wavelet detail $d_{3}$ coefficient \\
\hline$P_{32}$ & Noise of Wavelet detail $d_{4}$ coefficient \\
\hline
\end{tabular}

Equation (2) represents the entire matrix for ' $m$ ' whistlers. Each whistlers has 32 features extracted and hence the each input matrix when training with ' $\mathrm{m}$ ' signals simultaneously would be $32 \times \mathrm{m}$.

$$
F_{\text {input }}=\left[\begin{array}{ccccccc}
P_{1,1} & P_{1,2} & P_{1,3} & P_{1,4} & & P_{1, m-1} & P_{1, m} \\
P_{2,1} & P_{2,2} & P_{2,3} & P_{2,4} & & P_{2, m-1} & P_{2, m} \\
P_{3,1} & P_{3,2} & P_{3,3} & P_{3,4} & \ldots & P_{3, m-1} & P_{3, m} \\
\vdots & \vdots & \vdots & \vdots & \vdots & \vdots & \vdots \\
P_{30,1} & P_{30,2} & P_{30,3} & P_{30,4} & \cdots & P_{30, m-1} & P_{30, m} \\
P_{31,1} & P_{31,2} & P_{31,3} & P_{31,4} & & P_{31, m-1} & P_{31, m} \\
P_{32,1} & P_{32,2} & P_{32,3} & P_{32,4} & & P_{32, m-1} & P_{32, m}
\end{array}\right]
$$

\section{Neural network architecture}

The most common type of NN called Feed-Forward Multi-layer perceptron [23]along withLevenberg-Marquardt algorithm based on the two layer perceptron as illustrated in Fig. 2 was used in this work. 


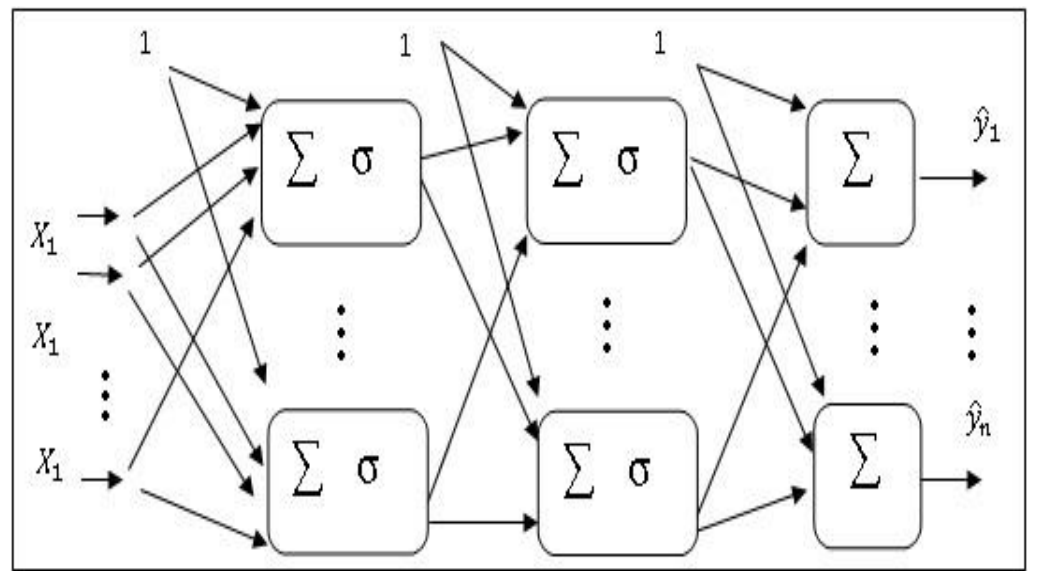

Fig. 2.Two layer perceptron of Levenberg-Marquardt neural network applied to identify to the whistlers.

The output layer has one node for target whistler. There are no hard and fast rules for choosing the number of hidden layers and the number of nodes in each of the hidden layers. It is generally believed that one hidden layer is sufficient for any network architecture [24, 25]. Many authors established the use of one hidden layer for successful trained NN $[26,27,28]$. Several authors have employed NNs with more than one hidden layer based on the complexity of their networks [29, 30, 31, 32]. This type of a neural network is known as 'supervised'. This involves two main stages of solving a problem; the training of the network and the recognition (the prediction itself). In supervised learning scheme the network is taught the relationship between the input and output pairs, which is called the training set.

To train the neural network, we created teaching databases using the observations of DEMETER satellite form January 2008 to December 2009. The optimal properties for the formation of teaching database were derived after many experiments on teaching and testing of neural networks. As a result, the training samples included the various features calculated form Statistical and Wavelet based techniques. The neural network performance strongly depends on the selection of training data set. Proposed FFNN has been trained with 160 samples of each whistler. To achieve good responses the whole data set was randomly divided in to two independent data sets known as training (70\%) and testing (30\%) for training and testing purpose [25]. The training set (TR) contained the data used to update the synaptic weights while the testing data set is used to check that the $\mathrm{NN}$ is not being over-trained. This process is terminated when the test error values versus the number of training epochs pass through a predetermined amount [33].

\section{- Performance analysis}

\section{Results}

In order to find best FFNN for whistler identification, we trained several different NN with different architectures. The best NN architecture obtained in this case was a NN with 5 units and 1000 epochs. The choice of this NN is based on its better performance in terms of Mean Square Error (MSE) and regression analysis, when compared with the results of other configurations. It should be clear that the choice of number of nodes for each hidden layer were determined on a trial and error basis. The transig transfer with weight between -1 and 1 was applied as an activation function.

\section{- Mean Square Error (MSE)}

The MSE is computed by taking the differences between the target and the actual NN output, squaring them and averaging over all classes and internal validation samples. Analytically, it is given by

$$
M S E=\frac{1}{p} \sum_{p}\left(t_{p}-o_{p}\right)(3)
$$

where number of input-output training pairs is represented by $\mathrm{p}, \mathrm{p}^{\text {th }}$ training for the target output is represented by $t_{p}$ ando $o_{p}$ is the output of FFNN. The variation of system error rate with respect to the error number during training iterations is shown in Fig. 3. It was noticed that, the results are stable and no fluctuations are observed. MSE of 0.001 was observed for our optimized FFNN with a training MSE $10^{-12}$. 


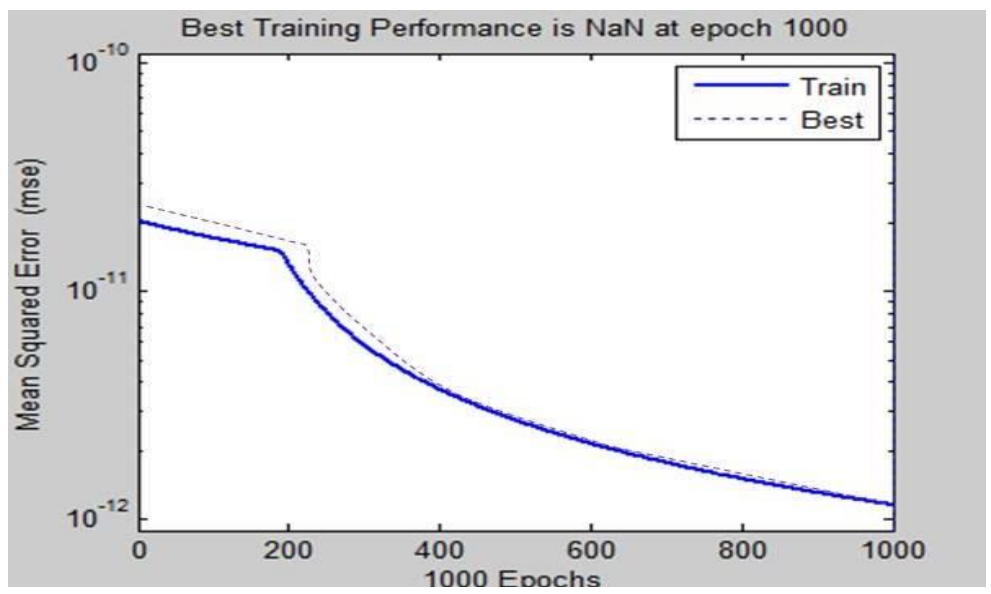

Fig. 3. Mean Square plot of proposed FFNN Blue line represents the MSE for training and dotted line is line of

\section{- Regression analysis} best fit.

The output of regression model is

$$
y=X_{w}+\varepsilon
$$

Here $\mathrm{y}$ is the output vector; $\mathrm{X}$ is the data matrix, $w$ is the parameter vector and $\varepsilon$ is the error vector. In a regression plot (see Fig. 4) a perfect correlation between the predicted and targets was obtained as indicated by the solid line. The dashed line indicates the best fit produced by the algorithm. The value of correlation coefficient $\mathrm{R}$ is equal to 1 .

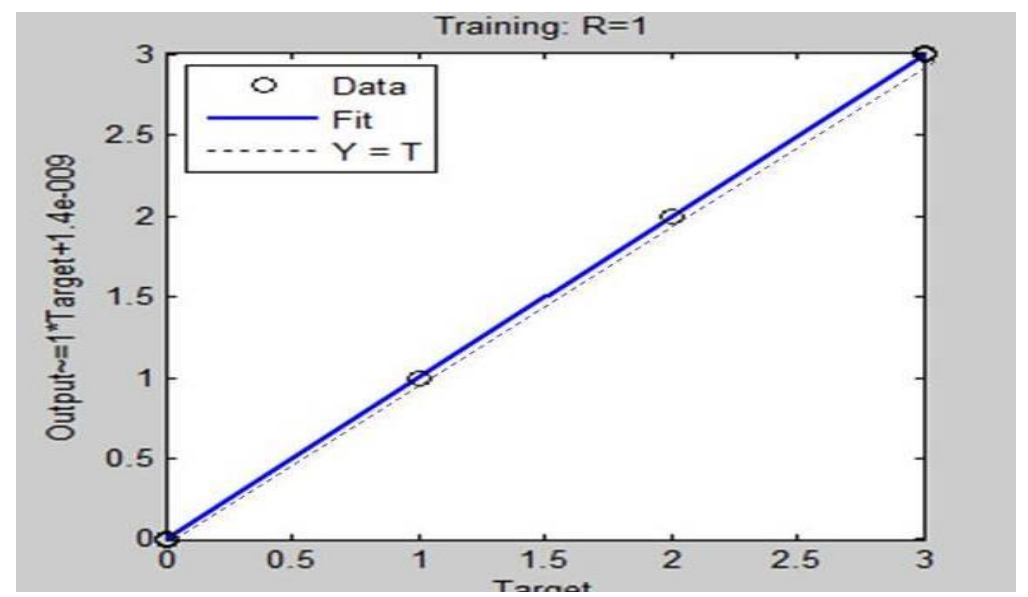

Fig. 4.Regression plot for proposed Feed Forward Neural Network (FFNN).

\section{Demeter satellite data analysis}

We have used four types of whistlers (i.edispersive, multipath, spiky and diffuse) for the training. Each group consists of 160 samples of slightly different characteristics. Each sample was in a vector form with 32 components representing 32 features extracted from the proposed feature extraction technique. These features are used to train the FFNN. After the training test signals were used to verify the performance of the NN. As an example, the overall scheme including wavelet feature extraction and identification is shown in Fig. $5(\mathrm{a}, \mathrm{b})$ for VLF signals recorded at 9:56:52:252 to 9:56:52:694 UT and 9:07:46:187 to 9:07:46:383 UT on January 01, 2008 by DEMETER satellite. This shows that the identified whistler was multipath whistler in Fig. 5 (a) and dispersive whistler in Fig. 5 (b). The results of NN identifier are reported in Table 2. As can be seen from the Table 2, proposed FFNN perform quite well and correctly identify whistler from the observed VLF signal. The most significant performance is found for Dispersive and spiky whistler with comparably less computational of $1.5 \mathrm{sec}$ and overall accuracy of $99 \%$ with $1 \%$ error rate. 


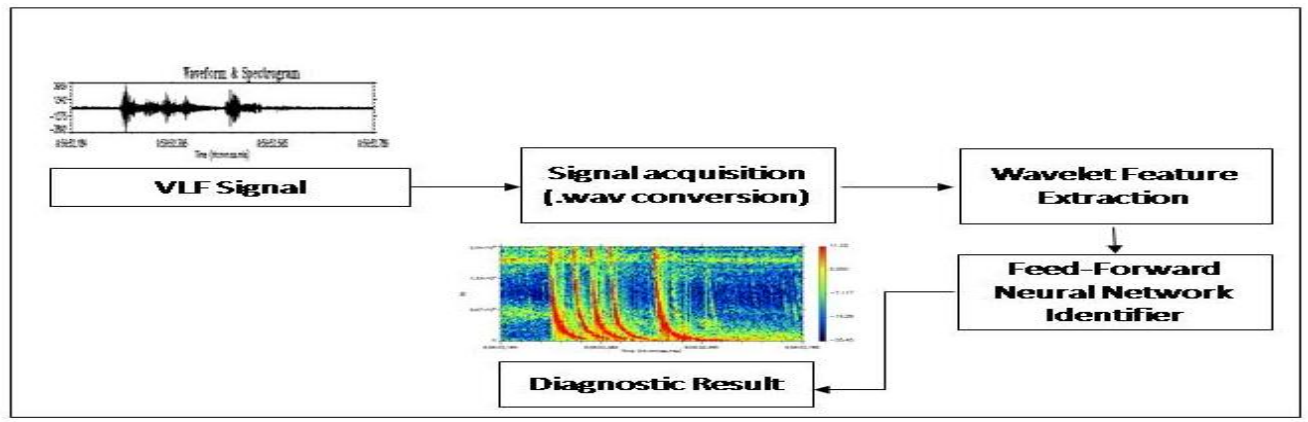

Fig. 5 (a). Identification of multipath whistler form VLF signal recorded at 9:56:52:252 to 9:56:52:694 UT, Orbit 19094 (up) on January 01, 2008, by DEMETER satellite.

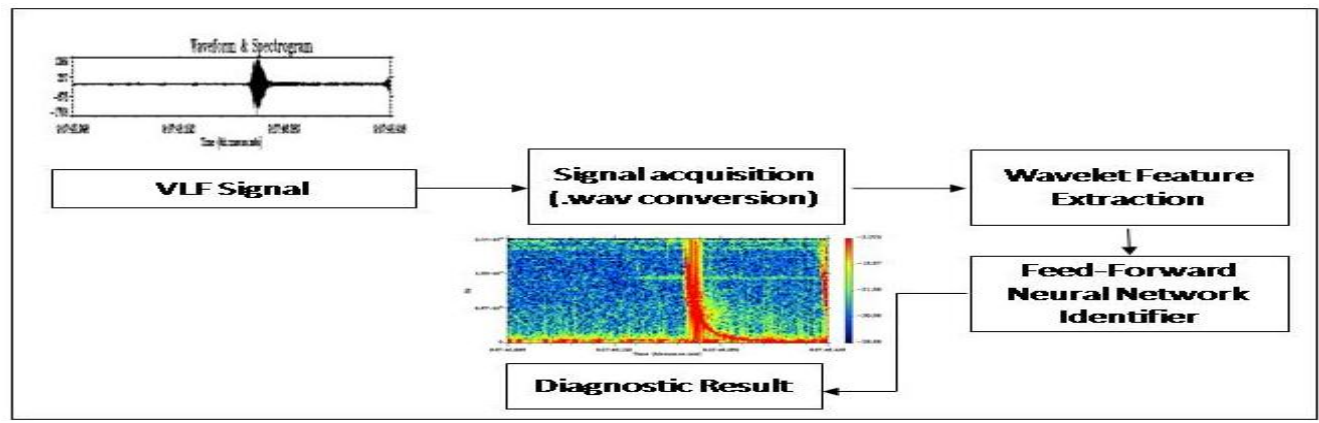

Fig. 5 (b). Identification of dispersive whistler form VLF signal recorded at 9:07:46:187 to 9:07:46:383 UT, Orbit 19094 (down) on January 01, 2008 by DEMETER satellite.

Table 2.Performance of proposed Feed Forward Neural Network (FFNN) identifier

\begin{tabular}{clccc}
\hline Whistlers & Algorithm & Computational Time & Performance & Error \\
\hline Diffuse & Levenberg-Marquardt & $2 \mathrm{sec}$ & $97 \%$ & $3 \%$ \\
Dispersive & Levenberg-Marquardt & $1.5 \mathrm{sec}$ & $99 \%$ & $1 \%$ \\
Multipath & Levenberg-Marquardt & $2.5 \mathrm{sec}$ & $98 \%$ & $2 \%$ \\
Spiky & Levenberg-Marquardt & $1.5 \mathrm{sec}$ & $99 \%$ & $1 \%$ \\
\hline
\end{tabular}

\section{Discussion and Conclusion}

The objective of this paper is to develop a Feed Forward Neural Network (FFNN) based on Levenberg-Marquardt learning algorithms for the identification of whistlers. Standard Levenberg-Marquardt algorithms take comparably less computing time as compared to sigmoid implementation with ADSP-21020 component [21] and iteration computing for good performance and less accepted error rate. A sequence of statistics and Wavelet transform based features proved useful for whistler identification. The Wavelet scale energy noise represents information about the behavior of whistler at different time scales. Schmitter[34] also showed that Wavelet and Statistical based features are very efficient for the analysis and characterization of transients and input into a radial basis function Neural Network that is train to discriminate transients (i.e., pulse like to wave like sferic). To evaluate this features, we simulated some cases of identification data and different setting of network factors (e.g., hidden layer number and nodes, number of classification and interaction) with same conditions. Despite some mis-identification errors, the proposed automatic identifier shows good overall accuracy greater than $97 \%$ for all whistler. This result is more pronounced as compare to previous study done by Miniere et al. [20]. They produced a time delay NN for identification of fractional and proton whistlers. In the best case it give $85.5 \%$ recognition performance with 4 neutrons in the first hidden layer and 3 neurons in the second hidden layer.

The trained FFNN's capability of immediately providing reliable identification of whistler, which can be profitably used to study the ionospheric perturbations, hereafter, can be applied to study the effect of seismic and volcanic activity over the ionosphere. Popova et al. [35, 36] used NN for the prediction of seismic events based on low frequency signal monitored at Kuril-Kamcharka and Japanese region. The results confirm that prediction of earthquake based on changes in LF signals is possible. Picchiani et al. [37] proposed a NN for the classification of volcanic plumes in a cloudy scenario with overall accuracy of $84 \%$. Furthermore to improve the FFNN's accuracy, additional data can be considered. However, it is not necessary if the training set is a perfect representative of the studied whistler. Further, development could enhance the FFNN's potentials by improving its capability to identify the other types of whistler transients. At this time we still continue to study new discriminating features that could be efficiently implemented to allow real-time computation in order to increase the power of onboard identification system. 


\section{Acknowledgements}

The authors wish to thanks DEMETER satellite mission center, CNES France for providing data required for this work.

\section{References}

[1] R.A. Helliwell, Whistlers and Related Ionospheric Phenomena, (Stanford University Press, Stanford, USA, 1965).

[2] D.L. Carpenter and R.L. Smith, Whistler measurements of electron density in the magnetosphere, Reviews of Geophysics, 2, 1964, $415-441$.

[3] R.P. Singh, A.K. Singh, and D.K. Singh, Plasmaspheric parameters as determined from whistler spectrograms: a review,Journal of Atmospheric and Solar - Terrestrial Physics, 60, 1998, 495 - 508.

[4] D.L. Carpenter andC. Park, Pioneer of magnetosphere ionosphere coupling research,Journal of Atmospheric and Solar-Terrestrial Physics, 69, 2007, $351-361$.

[5] M.J. LeDocq, D.A. Gurnett and R.R. Anderson, Electron number density fluctuations near the plasmapause observed by the CRRES spacecraft, Journal of Geophysical Research, 99 (23), 1994, 661.

[6] J. Bortnik, U.S. Inan, and T.F. Bell, Frequency-time spectra of magnetospherically reflecting whistlers intheplasmasphere, Journal ofGeophysical. Research, 108 (A1), 2003, 1030.

[7] J.Y. Liu, K. Wang, C.H. Chen, W.H. Yang, Y.H. Yen, Y.I. Chen, K. Hattori, H.T. Su, R.K. Hsu, C.H. Chang,A Statistical study in ELF-whistlers/emission and M>5.0 earthquake in Taiwan, Journal of Geophysical Research, 118 (6), 2013,3760 - 3768.

[8] J. Błecki, M. Kosciesza, M. Parrot, S. Savin, and R. Wronowski, Extremely low frequency plasma turbulence recorded by the DEMETER satellite in the ionosphere over the Abruzzi region prior to the April 6, 2009, L'Aquila earthquake, Annals of Geophysics, 55 (1), 2012, $37-47$

[9] M.E. Murat, and A.J. Rudman, Automated first arrival picking: A neural network approach, Geophys. Prosp., $40,1992,587$ - 604.

[10] M.M. Poulton, B.K. Sternberg and C.E. Glass, Location of subsurface targets in geophysical data using neural networks. Geophysics, 57, 1992, $1534-1544$

[11] H. Langer, G. Nunnari and L. Occhipinti, Estimation of seismic waveform governing parameters with neural networks, Journal of Geophysical Research, 101(20), 1996,109 - 118.

[12] C. Calder 'on-Mac'ias, M.K. Sen, and P.L. Stoffa, Hopfield neural networks, and mean field annealing for seismic deconvolution and multiple attenuation, Geophysics, 62, 1997, 992 - 1002.

[13] L.X. Wang and J.M. Mendel, Adaptive minimum predictionerrordeconvolution and source wavelet estimation using Hopfield neural networks. Geophysics, 57, 1992, 670 - 679.

[14] F.U. Dowla, S.R. Taylor, and R.W. Anderson, Seismic discrimination with artificial neural networks: Preliminary results with regional spectral data, The Bulletin of the Seismological Society of America, 80, 1990, 1346 - 1373.

[15] G. Romeo, Seismic signals detection and classification using artificial neural networks, Annali di Geofisica, 37, $1994,343-353$.

[16] E.D. Schmitter, Characterization and Classification of Natural Transients, International Journal of Environmental, Ecological, Geological and Geophysical Engineering, 2 (1), 2006, 201 - 213.

[17] A. Waibel, T.Hanazawa and G. Hinton, Phoneme recognition using Time-delay Neural Network, IEEE transaction on Acoustics, Speech and signal processing, 37 (3), 1989, $328-339$.

[18] A. Buzzi,Demeter Satellite Data Analysis of Seismo-Electromagnetic Signals, Doctorate Thesis, Roma Tre University of Rome, 2004.

[19] I. Popova, A. Rozhnoi, M.Solovieva, B. Levin, M. Hayakawa, P.F. Biagi and K. Schwingenschuh, Application of Neural Network Methodology in Analysis of VLF/LF Signal, Abstract 1-09, GotembaKogen Resort, Gotemba, Japan October 1-4, 2012.

[20] X. Miniere, J. Pincon, and F.Lefeure, A neural network approach to the classification of electron and proton whistlers,Journal of Atmospheric and Terrestrial Physics, 58, (7), 1996, 911 - 924

[21] F. Elie, M. Hayakawa, M. Parrot, J. Pincon and F. Lefeuvre, Neural network system for the analysis of transient phenomena on board the DEMETER micro-satellite, IEICE Trans Fundamentals. E82(8), 1999, 1575-1581.

[22] S. Mallat, A Wavelet Tour of Signal Processing 2ed, (Academic Press, 1999).

[23] C.M. Bishop, Neural Networks for Pattern Recognition (Oxford University Press, New York, 1995).

[24] L.V. Fausett, Fundamentals of neural networks: Architecture, algorithms, and applications (Prentice-Hall, Englewood Cliffs, 1994).

[25] S. Haykin,Neural Networks: A Comprehensive Foundation(New York: MacMillan Publishing Company, 1994).

[26] L.A. Williscroft and A.W.V. Poole, Neural networks, foF2, sunspot number and magnetic activity, Geophysical. Research Letter, 23(24), 1996, 3659 - 3662 .

[27] Y. Tulunay, E. Tulunay and E.T. Senalp, An attempt to model the influence of the trough on HF communication by using neural network, Radio Science, 36(5), 2001, 1027 - 1041.

[28] A.W.V. Poole and M. Poole, Long-term trends in foF2 over Grahamstown using neural networks, Annals of Geophysics, 45, 2002, $155-161$.

[29] Y. Hirose, K. Yamashita and S. Hijiya, Back-propagation algorithm which varies the number of hidden units,Neural Networks, 4,1991, 61-66

[30] X. Lamming and L.R. Cander, Monthly median foF2 modelling COST 251 Area by neural networks, Physics and Chemistry of the Earth, 24, 1999, 349 - 354

[31] L. Derong, E. Myron, Hohilb, H. Stanley and Smithc, N-bit parity neural networks: New solutions based on linear programming, Neuro computing, 48, 2002, 477 - 488.

[32] Th.D. Xenos, P.Spalla and C. Mitchel, Neural network based TEC forecasting models, AP-2000 Meeting at Davos, Switzerland, 2000 .

[33] A. Kumluca, E. Tulunay, I. Topalli and Y.Tulunay, Temporal and spatial forecasting of ionospheric critical frequency using neural networks, Radio Science, 34, 1999, 1497 - 1506

[34] E.D. Schmitter, Analysing and Classifying VLF Transients, International Journal of Signal Processing, 3(4), 2007,238 -242.

[35] I. Popova, A.Rozhnoi, M.Solovieva, B. Levin, M. Hayakawa, Y. YasuhideY.Hobara, P. Biagi, and K. Schwingenschuh, Neural network approach to the prediction of seismic events based on low-frequency signal monitoring of the Kuril-Kamchatka and Japanese regions, Annals of Geophysics, 2013, 56 (3), 2013, 1 -7

[36] I. Popova, A. Rozhnoi, M. Solovieva, B. Levin, and V. Chebrov, Neural network technique for identifying prognostic anomalies from low-frequency electromagnetic signals in the Kuril-Kamchatka region, Physics of the Solid Earth, 52 (2),2016, $305-317$.

[37] M. Picchiani, M.Chini, S.Corradini, L. Merucci, A. Piscini and F.D. Frate, Neural Network Multispectral Satellite Images Classification of Volcanic Ash Plumes In A Cloudy Scenario, Annals Of Geophysics, Fast Track 2, 2014. 